\title{
CHARA Michigan phase-tracker (CHAMP): a preliminary performance report
}

David H. Berger, John D. Monnier, Rafael Millan-Gabet, Theo A. ten Brummelaar, Matthew Anderson, et al.

David H. Berger, John D. Monnier, Rafael Millan-Gabet, Theo A. ten Brummelaar, Matthew Anderson, Jennifer L. Blum, Timothy Blasius, Ettore Pedretti, Nathalie Thureau, "CHARA Michigan phase-tracker (CHAMP): a preliminary performance report," Proc. SPIE 7013, Optical and Infrared Interferometry, 701319 (28 July 2008); doi: 10.1117/12.790168

Event: SPIE Astronomical Telescopes + Instrumentation, 2008, Marseille, France 


\title{
CHARA Michigan Phase-tracker (CHAMP): A preliminary performance report
}

\author{
David H. Berger ${ }^{a}$, John D. Monnier ${ }^{a}$, Rafael Millan-Gabet ${ }^{b}$, Theo A. ten Brummelaar $^{c}$, \\ Matthew Anderson ${ }^{a}$, Jennifer L. Blum ${ }^{a}$, Timothy Blasius ${ }^{a}$, Ettore Pedretti ${ }^{d}$, and Nathalie \\ Thureau $^{d}$ \\ ${ }^{a}$ University of Michigan, Department of Astronomy, 500 Church St, Ann Arbor, MI 48109, \\ USA; \\ ${ }^{b}$ Michelson Science Center, California Institute of Technology, 770 South Wilson Avenue, \\ Pasadena, CA 91125, USA; \\ ${ }^{c}$ The CHARA Array, Mount Wilson Observatory, Mount Wilson, CA 91023, USA; \\ ${ }^{d}$ School of Physics and Astronomy University of St Andrews, St Andrews, Scotland, UK;
}

\begin{abstract}
The CHARA Michigan Phase-tracker (CHAMP) is a real-time fringe tracker for the CHARA Array, a sixtelescope long baseline optical interferometer on Mount Wilson, California. CHAMP has been optimized for tracking sensitivity at $\mathrm{J}, \mathrm{H}$, or $\mathrm{K}$ bands and is not meant as a science instrument itself. This ultimately results in maximum sensitivity for all the science beam combiners that benefit from stabilized fringes. CHAMP was designed, built, and tested in the laboratory at the University of Michigan and will be delivered to the CHARA Array in 2008. We present the final design of CHAMP, highlighting some its key characteristics, including a novel post-combination transport and imaging system. We also discuss testing and validation studies and present first closed-loop operation in the laboratory.
\end{abstract}

Keywords: long baseline optical interferometry; fringe tracking; near infrared; instrumentation

\section{INTRODUCTION}

This paper is a follow-up to the preliminary description of the CHARA Michigan Phase-tracker (CHAMP) in Ref. 1 (henceforth Paper I). CHAMP is the high-bandwidth fringe tracker for the Center for High Angular Resolution Astronomy (CHARA) Array, a six-telescope long-baseline optical interferometer on Mount Wilson, California. It will enable coherent integration capabilities for the science instruments, specifically the Michigan InfraRed Combiner (MIRC), ${ }^{2,3}$ by sensing atmospherically induced phase fluctuations of the fringe signal and providing corrections to the optical delay lines.

Currently, fringes at the CHARA Array are detected by the individual science instruments. Each combiner ("Classic", ${ }^{4}$ FLUOR,${ }^{5}$ VEGA* ${ }^{*} \mathrm{PAVO}^{\dagger}$, and MIRC) uses some variant of group delay or "packet" tracking to follow atmospheric turbulence within a coherence length, usually at low bandwidth $(<5 \mathrm{~Hz})$ On nights of good seeing, this amounts to optical path fluctuations of tens of microns or more within 100-1000 ms (on poor seeing nights, the fluctuations are 10-100 $\times$ faster).

We have adopted the philosophy that a fringe tracker should be separate from the science instruments, an idea that was part of the original design plan for the CHARA Array ${ }^{6}$ and is reminiscent of the design approach of modern adaptive optics systems on large-aperture telescopes. Under this construct, the fringe tracker and science combiners can be uniquely optimized and independently developed. The task of measuring well-calibrated, high precision visibilities and/or closure phases is therefore left to the science instrument, and the fringe tracker can be

Further author information: (Send correspondence to D.H.B.)

D.H.B.: E-mail: dhberger@umich.edu, Telephone: 17347644160

*Mourard et al., these proceedings

${ }^{\dagger}$ Ireland et al., these proceedings

Optical and Infrared Interferometry, edited by Markus Schöller, William C. Danchi, Françoise Delplancke Proc. of SPIE Vol. 7013, 701319, (2008) · 0277-786X/08/\$18 - doi: 10.1117/12.790168 


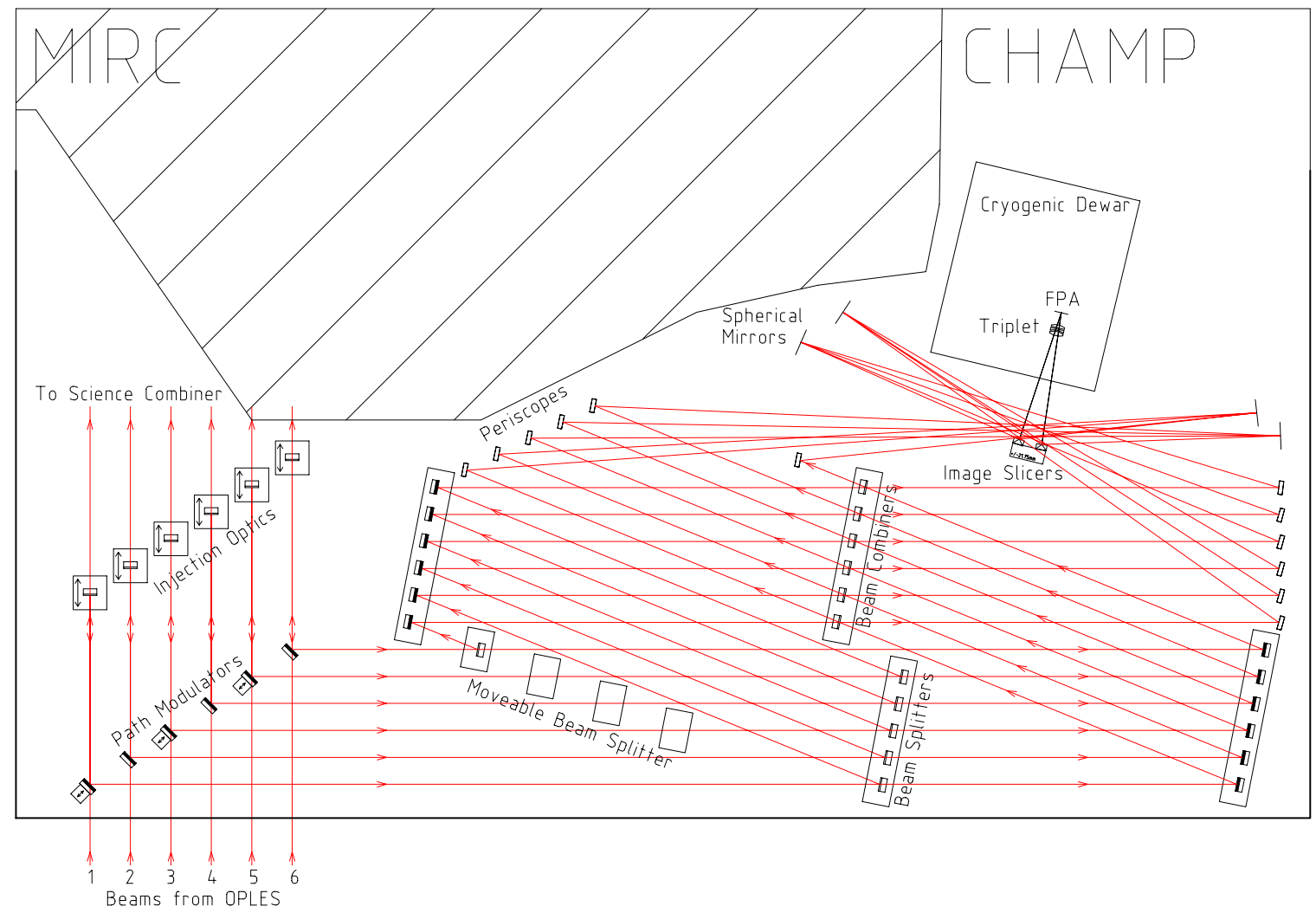

Figure 1. The CHAMP optical layout. Note that injection optics are at a 6" beam height and the remaining CHAMP path modulators and beam combiners are at a 4.5 " beam height. Details of each system are in Section 2 . MIRC optics are not shown, but it footprint is shown as the hashed area.

designed primarily for increased sensitivity. The Very Large Telescope Interferometer (VLTI) has also adopted a similar philosophy for their fringetracker FINITO. ${ }^{7}$

Section 2 reviews the instrument layout and key components. While much of the overall design has remained the same since Paper I, several subsystems have been updated or redesigned. Specifically, the post-combination beam transport design has been changed from a fiber-based system to a bulk optics system. Also, the adopted readout pattern of the HAWAII-1 (FPA) is different than reported in Paper I, and the imaging optics inside the dewar have consequently been updated. In Section 3, we describe laboratory experiments aimed at verifying the system performance. Future work is discussed in Section 4.

\section{THE INSTRUMENT}

The CHARA Array will soon combine light from all six 1-meter telescopes simultaneously with separations ranging from 34 to 331 meters using the Michigan InfraRed Combiner (MIRC; Monnier et al., these proceedings) yielding a few tenths of a milliarcsecond resolution. As shown in Figure 1, MIRC and CHAMP occupy the same optical table, but CHAMP, as a service instrument, will provide six-beam, simultaneous phase estimates for all beam combiners. An overview of the CHAMP optical layout is described in Paper I, but is summarized here and an updated optical layout is illustrated in Figure 1. The six 20-mm diameter collimated IR beams approach the MIRC-CHAMP table spaced 3 inches apart having been path-length corrected and optionally compensated for longitudinal dispersion. ${ }^{8}$ 


\subsection{Injection and Combination}

Light from each near-IR beam is split between CHAMP and the science combiner. Beam splitters with custom dielectric coatings ${ }^{\ddagger}$ reflect either $\mathrm{J}+\mathrm{H}$-bands $(1.2$ to $1.7 \mu \mathrm{m})$, K-band $(2.0$ to $2.4 \mu \mathrm{m})$, or $50 \%$ intensity of all three bands into the CHAMP beam combiner. The complimentary portion of the beam in transmitted through to the science instrument(s). A choice of standard astronomical $\mathrm{J}, \mathrm{H}$, and $\mathrm{K}$ band filters ${ }^{\S}$ ultimately limit the light reaching the FPA.

The beams are injected into CHAMP by a $3^{\circ}$ downward reflection to elliptical fold mirrors at the 4.5" CHAMP beam height. Beams 1, 3, and 5 are path-modulated via the mirrors attached to piezoelectric actuators with an $8 \mu \mathrm{m}$ stroke. The beams are combined in a cyclical, pair-wise fashion $(1+2,2+3 \ldots 5+6,6+1)$ with the option for fewer beams to be used by moving a beam splitter. The beam combiner is a traditional Mach-Zehnder design containing two beam splitters and two fold mirrors per beam alleviating the need for a compensator plate. The six input beams yield twelve output beams, one pair per beam combiner.

\subsection{Post-Combination and Imaging Optics}

In Paper I, we described how the twelve output beams would be injected into fibers and re-imaged onto the center portion of a near-IR FPA, which we had assumed could be readout in a spiral-type pattern from the center. The HAWAII-1 FPA we purchased reads out from the corner of each quadrants starting at the outside edge and not the center. Consequently, in order to use the four quadrants in parallel, we must find a way to image the 12 output CHAMP beams into 4 bundles of 3 beams - each bundle separated by 512 pixels (one quadrant) in the image plane projected onto the FPA. This design requires a much wider field of view than originally envisioned. In order to resolve competing beam transport designs, our project held a design review in November 2006 and we outline the advantages and disadvantages of the possible post-combination systems here.

The first proposed design was the fiber transport system discussed in Paper I. The use of single-mode fibers has the strong advantage of not coupling excess infrared background into the cryogenic dewar; however, this design suffers from "false fringes" when used as a fringe tracker since tip-tilt tracking errors and vibrations cause fast intensity-coupling variations that can mimic fringe signals. In addition, single-mode fiber coupling is rarely optimal under realistic observing conditions. While standard multi-mode fibers would allow for more stable beam intensities, these fibers allow far too much infrared background into the system which would limit the K-band sensitivity. The compromise for an intermediate "many-mode" fiber was identified, but these fibers are not commercially produced and were not available with known performance characteristics. Lastly, maintaining internal cryogenic alignment of the fibers was deemed potentially either risky with no internal actuators or expensive with internal actuators that perform reliably at LN2 temperatures. There is also the operational difficulty in quickly optimizing the fiber coupling for 6 beams.

The second design proposed using bulk optics to focus the beams onto the FPA using 12 miniature beam trains that incorporate thermal baffling via individual cold pupils in the dewar. For this to be practical given the relatively small CHMAP dewar (and respecting the 3 inch vacuum window) the CHAMP beams would be reduced to $1 \mathrm{~cm}$ via either a monolithic or individual beam reducers to fit all twelve beams through window. After that, each beam would be spatially filtered and focused with small lenses inside the dewar. This design required many small lenses most of which would be inside the dewar. This design should be possible using modern fabrication techniques although the final focusing onto the detector quadrants was considered an open and difficult optical problem.

The third design also used bulk optics, but, instead of using a beam reducer, the beams are each focused to spots to an image plane located outside the dewar. The beams are then diverted using a kind of image-slicer so as to share a common cold pupil inside the dewar. Lastly, the external image plane is re-imaged onto the FPA with strong de-magnification using an achromatic triple. The advantages of this design are the ability to have a cold pupil inside of the dewar and a focus outside of the dewar for easy alignment. Lastly, only the triplet is inside the dewar, which greatly simplifies cryogenic alignment. The main difficulty proved to be the design and fabrication of the image-slicer optics. This design was the one selected, it has been fabricated, and we describe it more fully below.

\footnotetext{
${ }^{\ddagger}$ Omega Optical, Brattleboro, VT

${ }^{\S}$ Barr Associates, Westford, MA
} 


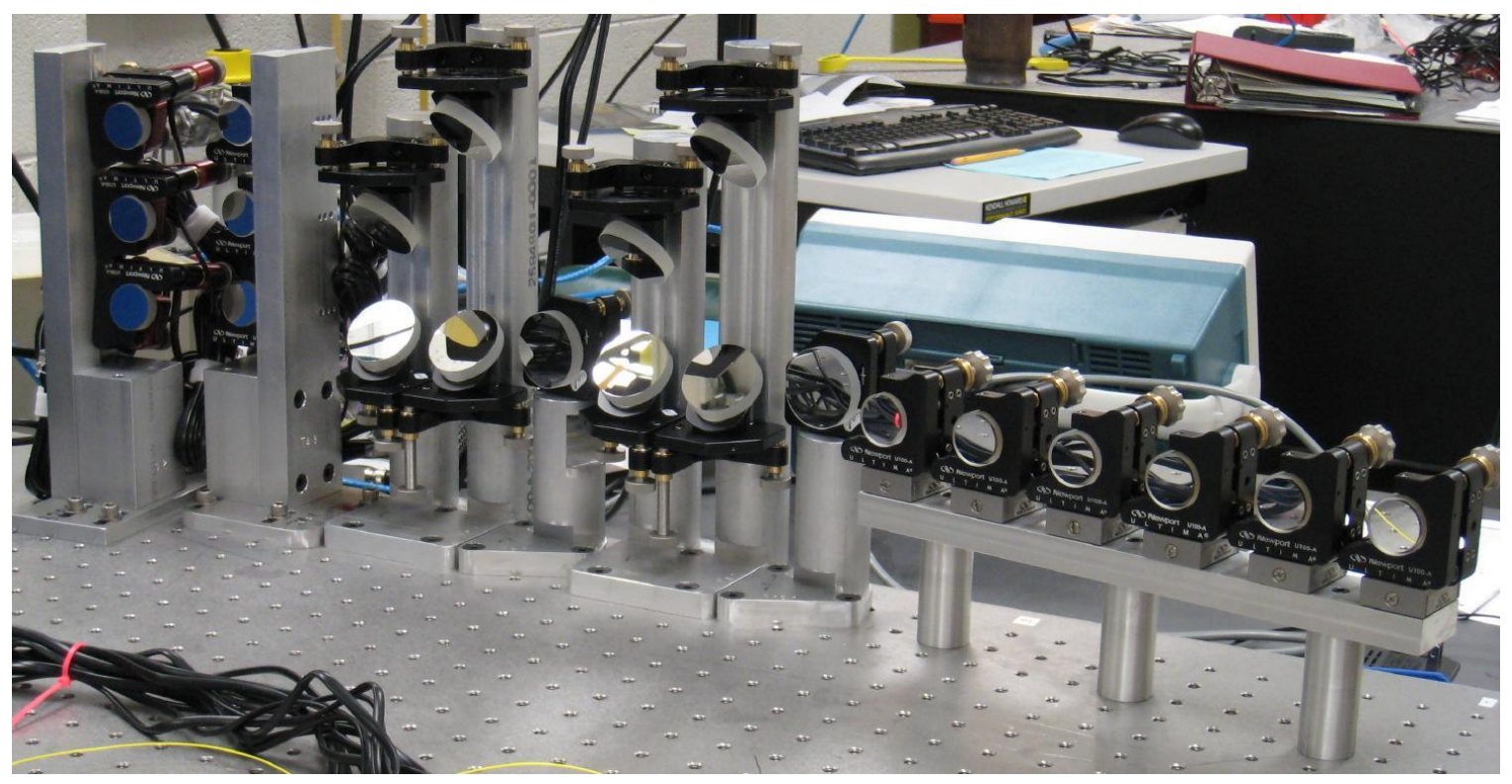

Figure 2. The right side of the CHAMP table as viewd in Figure 1. From left to right are the "Towers of Power", periscopes, and transmission-side fold mirrors.

\subsubsection{Spherical Mirrors}

To avoid expense and alignment difficulties, we chose to use spherical ( $\mathrm{R}=900 \mathrm{~mm}$, 1" diameter, ISP Optics) for creating the external image plane. In order to avoid spherical aberration, the incoming beams were $\mathrm{f} / 22$ and had to be used $\pm 2.5^{\circ}$ from the optical axis. This design led to the stacking of the mirrors vertically in an arrangement known as the "Tower of Power" (ToP - these are the only powered optics on the CHAMP table, except for the triplet inside the dewar). The CHAMP output beams are periscoped to different heights before being reflected to the ToPs. Figure 2 is a picture of right side of the CHAMP table as viewed in Figure 1 featuring the periscopes and spherical mirrors.

\subsubsection{Image Slicers}

The beams are focused onto the edge of a front-silvered prism, four of which are cemented together to form a pyramid (Figure 3$)$. This allows the spots to be placed very close together $(100 \mu \mathrm{m})$ while still permitting each beam to be directed to the common cold pupil. We use 4 pyramids, each one being brought to a focus at a different quadrant of the FPA. The base of the pyramids are 20x20 mm and have an apex height of $10 \mathrm{~mm}$. The pyramids were fabricated and assembled by Nu-Tek Precision Optical Corporation (Aberdeen, MD).

\subsubsection{Triplet}

As the twelve beams enter the cryogenic dewar, each beam is slowly diverging (f/22), but the beam paths from the separate pyramids are converging - all meeting just past the filter wheel at the $11 \mathrm{~mm}$ cold pupil. The triplet lens, initially designed by Anna Moore and Michael Ireland, is used to focus (achromatically) the beams onto the FPA such that each beam bundle is at the read-out corner and each beam is inside of one pixel. This requirement would normally mandate a large field of view (impossible to realize with only a triplet) but we were able to take advantage of the radial symmetry of the beam pattern on the FPA. By centering the optical axis in the middle of the readout corners, we were able to in essence reduce our corrected field-of-view to a tiny annulus - thus, optimization of the triplet was necessary in only the annulus and not over the entire FOV.

The lens triplet (fabricated by ISP Optics) is comprised of an IR-grade fused silica lens $\left(\mathrm{SiO}_{4}\right)$ between two $\mathrm{CaF}_{2}$ lenses. To minimize cost, two surfaces are polished flat and only two (the first and last) surfaces are aspherical. The $\mathrm{CaF}_{2}$ lenses were diamond-turned at small additional cost. To facilitate mounting, the outer 5 $\mathrm{mm}$ of each $25-\mathrm{mm}$ lens was left as a flat ledge. This is best seen in the picture of the lenses in Figure 4. Also 

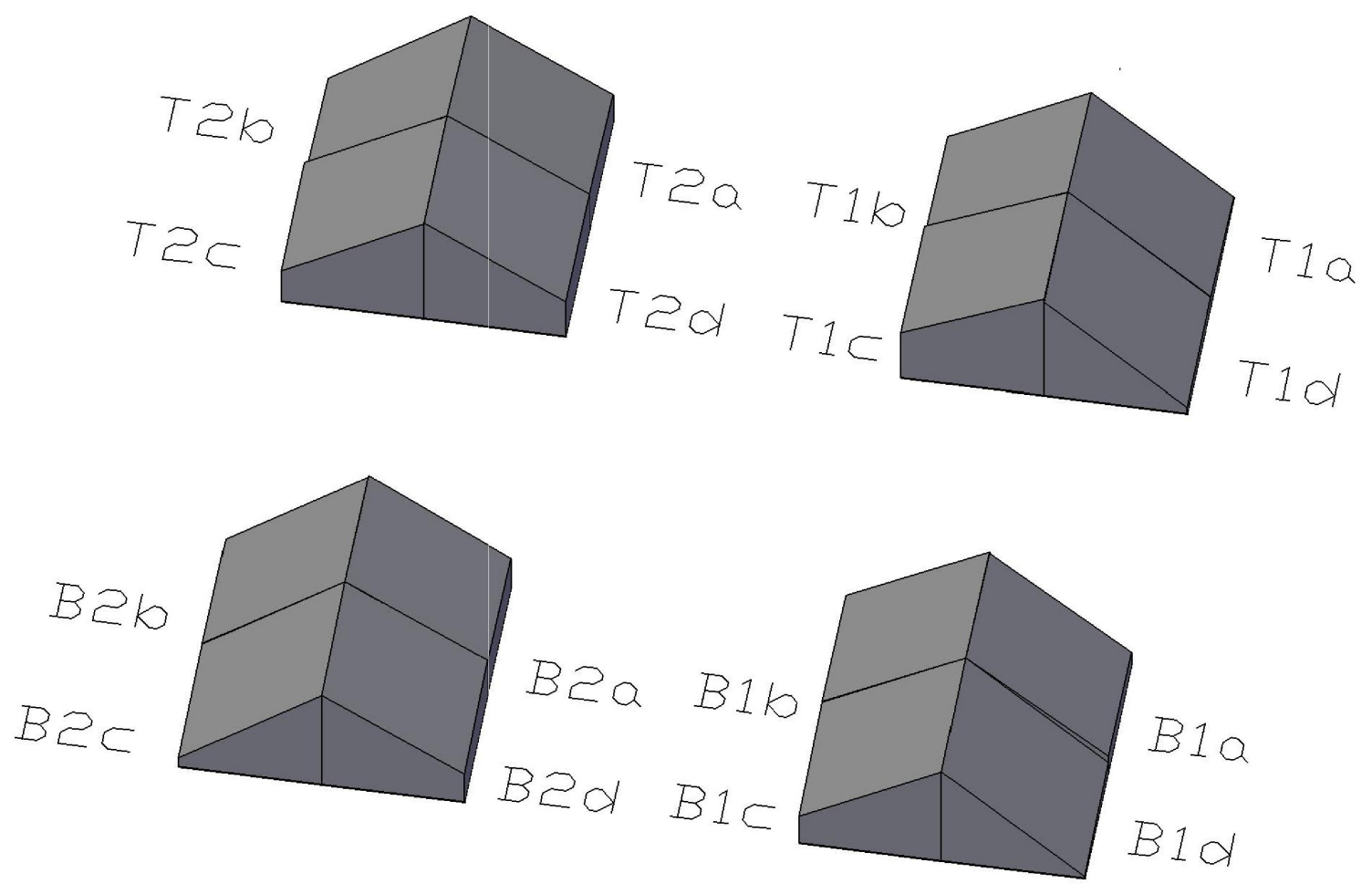

Figure 3. Silvered pyramid prisms are used as image slicers to divert close beams onto different quadrants of the focal plane array. The beams are forced to converge at a common cold pupil in the dewar for thermal baffling.

seen in the picture is the custom mount and spacer rings inside of the dewar, designed to accommodate varying thermal expansion coefficients of metal and glasses. Full testing of this system is yet to be performed.

In right panel of Figure 4, is the theoretical spot size at $\mathrm{J}, \mathrm{H}$, and $\mathrm{K}$ bands. Note that the triplet is achromatic over $\mathrm{J}, \mathrm{H}$, and $\mathrm{K}$ bands and the diffraction-limited spot size is smaller than the $18.5 \mu \mathrm{m}$ pixel size.

\subsection{Focal Plane Array, Electronics, and Computers}

The near-IR FPA is a HAWAII-1 , a 1024 x 1024 array of $18.5 \mu \mathrm{m}$ pixels split into four equal quadrants. It has a quantum efficiency of $>60 \%$ between 1 and $2.3 \mathrm{um}$, which overlaps with astronomical $\mathrm{J}, \mathrm{H}$, and $\mathrm{K}$ bands. Support electronics provided by Robert Leach (UCSB) contain the digital clocks and analog-to-digital converters. The digital clock signals are generated by a DSP and the four quadrants are readout simultaneously. The output from the FPA is an analog signal, which is amplified, digitized, and sent via a fiber optic cable to a custom PCI board installed in a PC running real-time linux (Gentoo/Xenomai).

A digital-to-analog converter (DAC) PCI card from National Instruments (PCI-6711) is connected to a multiport breakout box (BNC-2110") that delivers the analog signals to the amplifiers driving the piezo actuators. The modulation signal is pre-programmed onto the DAC and thus does not tap any resources from the computer during FPA readout. Synchronized with the modulation signal, the DAC also generates a TTL pulse to trigger the camera readout. The DSP code was customized to accommodate triggering and non-standard read-out/reset patterns.

Progress has also been made at CHARA upgrading the existing VMEbus computers and software to accept path-length offsets to the fast delay lines. Currently, the CHARA delay lines are controlled by three single board computers (MVME 1600s) and are limited to receiving offsets at $\sim 5 \mathrm{~Hz}$. These are being replaced by one single

\footnotetext{
ITeledyne Imaging Sensors, Thousand Oaks, CA

${ }^{\|}$National Instruments
} 


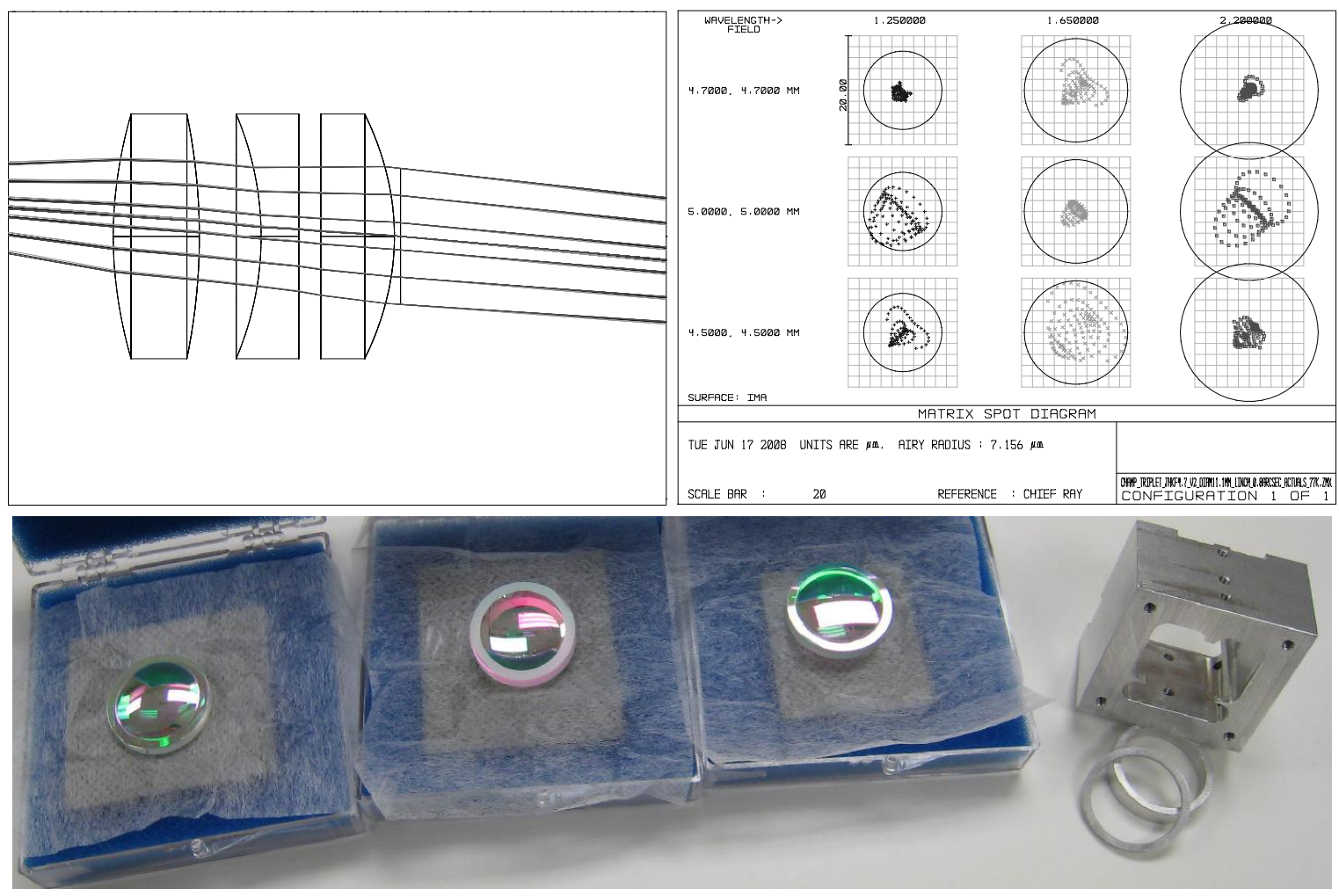

Figure 4. Clockwise from upper left: side view drawing of lenses and their spacing (image plane at detector on left, cold pupil shown as bar to the right of lenses), spot diagrams at J, H, and K bands, and a picture of the lenses and the custom mount and spacer rings.

board computer (Motorola MVME6100). Parallel to this, the host computer used to compile the delay line code has been changed from a Sun workstation to a Linux machine. Significant changes had to be made to the compiler and delay code to facilitate this upgrade. Much of this work has been done by Phil Irwin, who was part of the original team at JPL that first designed the delay lines for CHARA, PTI, and Keck Interferometer.

\section{PERFORMANCE VERIFICATION}

A distinct advantage to developing CHAMP in the laboratory at Michigan before shipping to CHARA was that we are able to characterize the system in a controlled environment. We present the results of our efforts to customize and synchronize the FPA read-out. Also included in our tests are the milestones of achieving white light fringes and subsequently closing the fringe-locking servo loop.

Figure 5 is a picture of CHAMP in the laboratory at Michigan. The light source is a collimated 20-mm beam split between two inputs of CHAMP. One arm of the injected beam can be adjusted by up to $200 \mu \mathrm{m}$ in path-length via a flat mirror attached to a piezoelectric actuator. The mirrors folding the beams into CHAMP can be moved to select any of the beam pairs. Only two adjacent beams can be combined at any one time, which is not a limitation at the CHARA Array. The beam splitter that divide the beams between the science combiner and CHAMP are also not used in this setup.

\subsection{Read noise}

As mentioned, the detector is a HAWAII-1 array detector, sensitive from 1 to $2.5 \mu \mathrm{m}$. In an effort to allow many non-destructive reads to reduce the effective read-noise, only a small part of each quadrant closest to the amplifier is read out. For our tests, we selected a $5 \times 5$ square of pixels, which allows for 1 beam per pixel with 1 pixel between each pixel. A mean-variance test showed that detector+electronics had a gain of $\sim 3.8$ 


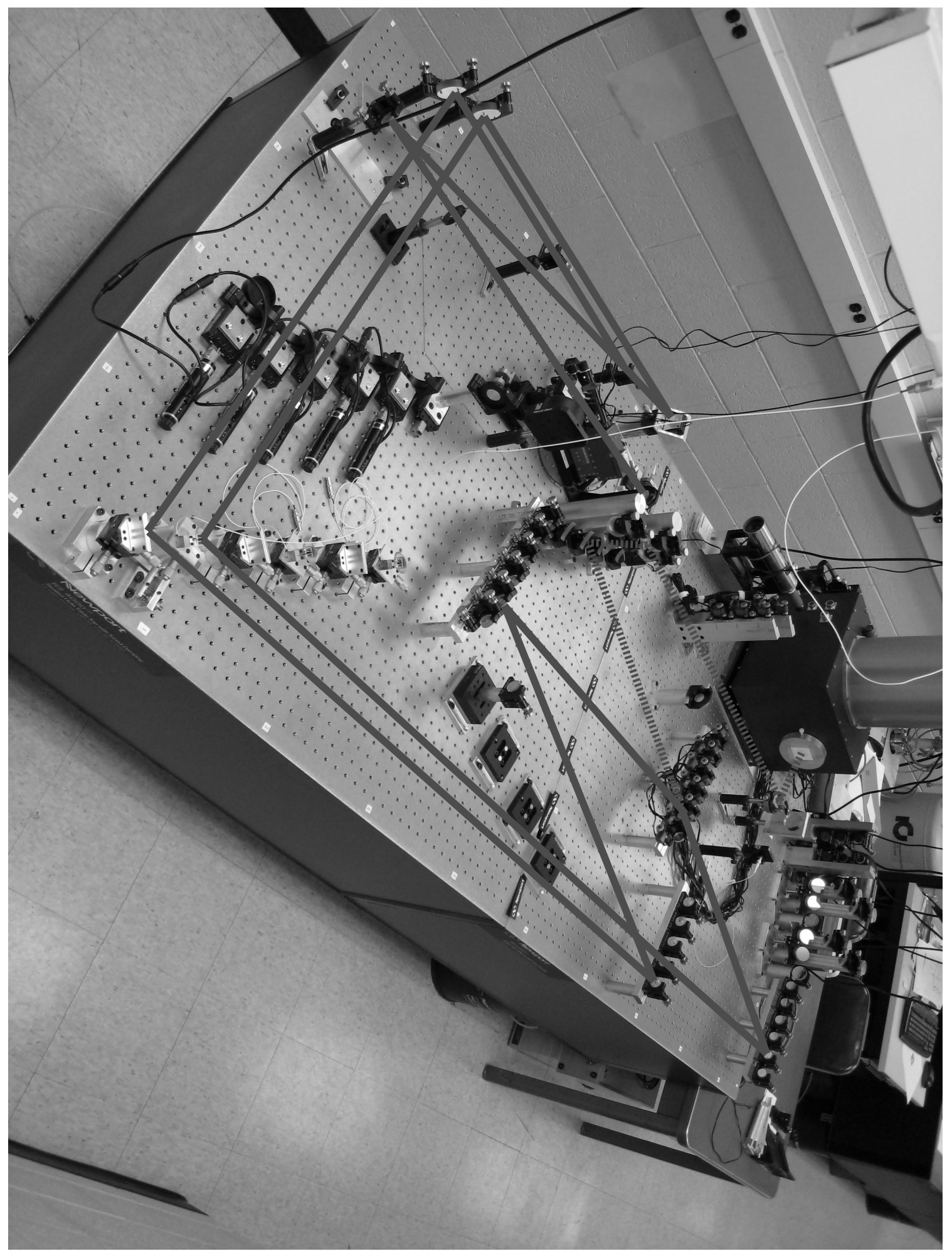

Figure 5. CHAMP in the laboratory at the University of Michigan. The drawn lines indicate the path of the white light beam from the source, through the beam combiner, and onto the detector. Dash lines indicate the beams after they have been combined. 


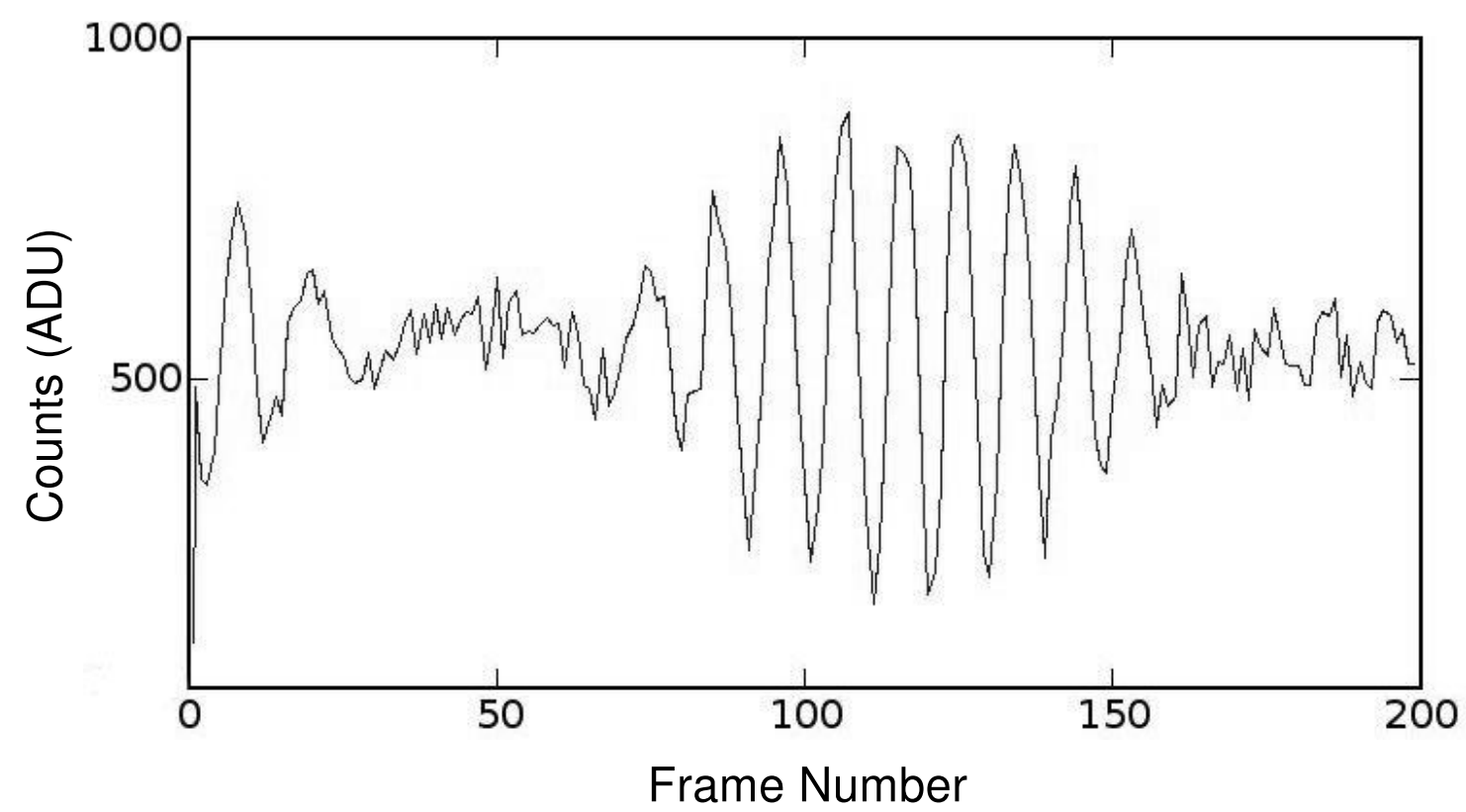

Figure 6. First white white fringes in the laboratory at Michigan. This represents 1 scan of approximately $40 \mu \mathrm{m}$ in length at H-band (1.65 um).

$\mathrm{e}^{-} /$ADU and $13 \mathrm{e}-$ read noise for using a single correlated-double sample (1 Mhz pixel clock). The DSP timing code was modified to allow for non-destructive reads and Fowler sampling which maximizes signal-to-noise ratio. However, we have discovered a $1 / \mathrm{f}$-noise source, which manifests as a noise floor of 6-10 $\mathrm{e}^{-}$(depending on the quadrant) even when reading each pixel 40 times. Further studies are being done to identity and eliminate this noise source which would restrict our J- and H-band tracking sensitivities (note at $\mathrm{K}$ band we expect to be thermal-background limited at for these read-noise values).

\subsection{White light fringes}

In early May 2008, we obtained white light fringes on beams 1 and 2 of CHAMP. The H-band (1.65 $\mu \mathrm{m})$ fringes shown in Figure 6 represent one scan of approximately $40 \mu \mathrm{m}$ in length using a long-scan piezo.

The white light source injects two beams with the same 11.2" phase delay between adjacent beams like at the CHARA Array. Therefore, CHAMP will be nominally phased with the in-situ science instruments upon delivery. Additionally, we have validated data acquisition synchronization between camera readout and path modulation.

\subsection{Closing the servo loop}

Shortly after obtaining white light fringes, we tested our preliminary fringe tracking algorithm. The laboratory setup was the same as in the previous section except path modulation was done with the short-stroke path modulators. Using a simple four-bin ( 5 sample) phase estimator, ${ }^{9}$ we measured the phase at a bandwidth of 62.5 Hz. Offsets were sent to a long-stroke piezo actuator that served as a "delay line". Figure 7 shows the measured phase with the servo off and then on for approximately 5 seconds each. Modulation was over $3 \mathrm{H}$-band fringes but only the middle one was used for phase estimation. With the servo loop closed, we achieved $0.1 \mathrm{rad}$ (26 $\mathrm{nm}$ ) RMS fringe lock. In operation, the phases of 3 to 5 fringes will be measured. The lock position will be the middle fringe, and the adjacent fringes will be used to measure group delay.

\section{FUTURE WORK}

Laboratory testing of CHAMP is nearly completed and shipment to the CHARA Array is expected by the end of Summer 2008. After integration, CHAMP will be commissioned on the sky followed by performance tests. Science with MIRC and CHAMP will commence in late 2008. 


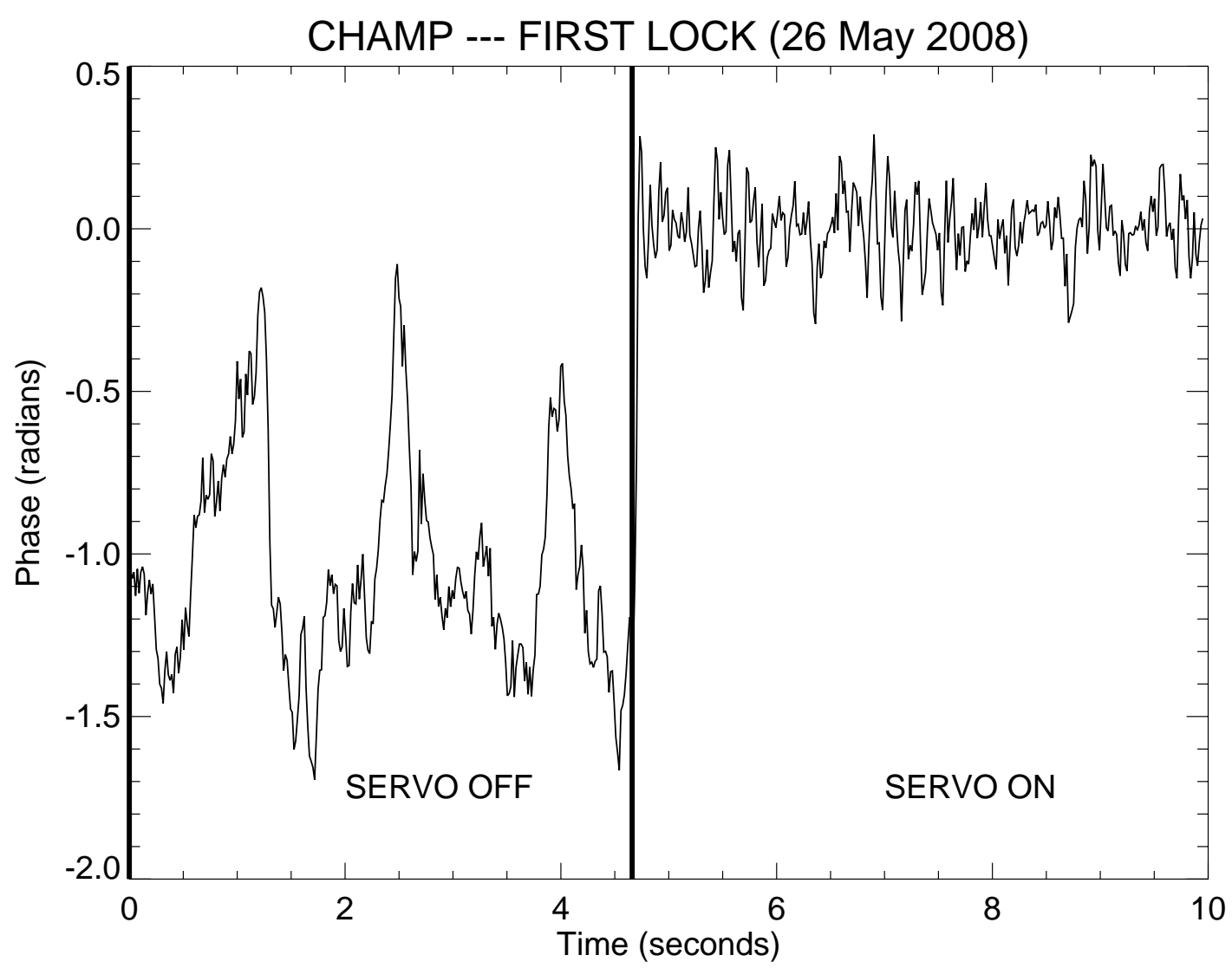

Figure 7. Fringe phase fluctuations due to laboratory seeing. The first 5 seconds are with the servo loop open (no offsets sent) and the last five seconds are with the servo loop closed.

\section{ACKNOWLEDGMENTS}

The fringe tracker funding, including financial support for DHB, was provided by the National Science Foundation through grant AST-0352723. We would like to thank Anna Moore and Michael Ireland for their initial design of the lens triplet. We would also like to thank Michael Ireland for consulting on many other aspects of CHAMP, especially the inspiration for the image slicer design. We acknowledge Scott Webster at the University of Michigan who single-handedly machined every custom part of CHAMP (all 126 parts and counting).

\section{REFERENCES}

[1] Berger, D. H., Monnier, J. D., Millan-Gabet, R., ten Brummelaar, T. A., Muirhead, P., Pedretti, E., and Thureau, N., "CHARA Michigan phase-tracker (CHAMP): design and fabrication," in [Advances in Stellar Interferometry. Edited by Monnier, John D.; Schöller, Markus; Danchi, William C.. Proceedings of the SPIE, Volume 6268, pp. 62683K (2006).], Presented at the Society of Photo-Optical Instrumentation Engineers (SPIE) Conference 6268 (July 2006).

[2] Monnier, J. D., Berger, J.-P., Millan-Gabet, R., and Ten Brummelaar, T. A., "The Michigan Infrared Combiner (MIRC): IR imaging with the CHARA Array," in [New Frontiers in Stellar Interferometry, Proceedings of SPIE Volume 5491. Edited by Wesley A. Traub. Bellingham, WA: The International Society for Optical Engineering, 2004., p.1370], Traub, W. A., ed., Presented at the Society of Photo-Optical Instrumentation Engineers (SPIE) Conference 5491, 1370-+ (Oct. 2004).

[3] Monnier, J. D., Pedretti, E., Thureau, N., Berger, J.-P., Millan-Gabet, R., ten Brummelaar, T., McAlister, H., Sturmann, J., Sturmann, L., Muirhead, P., Tannirkulam, A., Webster, S., and Zhao, M., "Michigan Infrared 
Combiner (MIRC): commissioning results at the CHARA Array," in [Advances in Stellar Interferometry. Edited by Monnier, John D.; Schöller, Markus; Danchi, William C.. Proceedings of the SPIE, Volume 6268, pp. 62681P (2006).], Presented at the Society of Photo-Optical Instrumentation Engineers (SPIE) Conference 6268 (July 2006).

[4] Sturmann, J., ten Brummelaar, T. A., Ridgway, S. T., Shure, M. A., Safizadeh, N., Sturmann, L., Turner, N. H. ., and McAlister, H. A., "Infrared beam combination at the CHARA array," in [Interferometry for Optical Astronomy II. Edited by Wesley A. Traub. Proceedings of the SPIE, Volume 4838, pp. 1208-1215 (2003).], Traub, W. A., ed., 1208-1215 (Feb. 2003).

[5] Mérand, A., Coudé du Foresto, V., Kellerer, A., ten Brummelaar, T., Reess, J.-M., and Ziegler, D., "CHARA/FLUOR updates and performance," in [Advances in Stellar Interferometry. Edited by Monnier, John D.; Schöller, Markus; Danchi, William C.. Proceedings of the SPIE, Volume 6268, pp. 62681F (2006).], Presented at the Society of Photo-Optical Instrumentation Engineers (SPIE) Conference 6268 (July 2006).

[6] ten Brummelaar, T. A., "Visible Light Fringe Tracking," tech. rep., Georgia State University/CHARA (1993). http://www.chara.gsu.edu/CHARA/Reports/appendixi.pdf.

[7] Gai, M., Menardi, S., Cesare, S., Bauvir, B., Bonino, D., Corcione, L., Dimmler, M., Massone, G., Reynaud, F., and Wallander, A., "The VLTI fringe sensors: FINITO and PRIMA FSU," in [New Frontiers in Stellar Interferometry, Proceedings of SPIE Volume 5491. Edited by Wesley A. Traub. Bellingham, WA: The International Society for Optical Engineering, 2004., p.528], Traub, W. A., ed., 528-+ (Oct. 2004).

[8] Berger, D. H., "Longitudinal Dispersion Compensation for a Long Baseline Optical Interferometer," PASP 116, 390-390 (Apr. 2004).

[9] Colavita, M. M., "Fringe Visibility Estimators for the Palomar Testbed Interferometer," PASP 111, 111-117 (Jan. 1999). 